\title{
A comprehensive simulation of weak-light phase-locking for space-borne gravitational wave antenna
}

\author{
DONG YuHui ${ }^{1,2 \dagger}$, LIU HeShan $^{1 \dagger}$, LUO ZiRen $^{3,4}$, LI YuQiong $^{1} \&$ JIN Gang $^{1 *}$ \\ ${ }^{1}$ National Microgravity Laboratory (NML), Institute of Mechanics, Chinese Academy of Sciences, Beijing 100190, China; \\ ${ }^{2}$ University of Chinese Academy of Sciences, Beijing 100190, China; \\ ${ }^{3}$ QUEST Centre of Quantum Engineering and Space-Time Research, Leibniz Universität Hannover, Hannover 30167, Germany; \\ ${ }^{4}$ Max-Planck-Institude for Gravitational Physics (Albert Einstein Institute), Hannover 30167, Germany
}

Received January 8, 2016; accepted March 11, 2016; published online April 7, 2016

\begin{abstract}
A comprehensive simulation was performed to better understand the impacts and effects of the additional technical noises on weak-light phase-locking for LISA. The result showed that the phase of the slave laser tracked well with the received transmitting light under different noise level, and the locking precision was limited by the phase readout noise when the laser frequency noise and clock jitter noise were removed. This result was then confirmed by a benchtop experimental test. The required LISA noise floor was recovered from the simulation which proved the validity of the simulation program. In order to convert the noise function into real time data with random characteristics, an algorism based on Fourier transform was also invented.
\end{abstract}

weak-light phase-locking, gravitational wave detection, heterodyne laser interferometer

Citation: Dong Y H, Liu H S, Luo Z R, et al. A comprehensive simulation of weak-light phase-locking for space-borne gravitational wave antenna. Sci China Tech Sci, 2016, 59: 730-737, doi: 10.1007/s11431-016-6043-0

\section{Introduction}

The space-borne gravitational wave (G.W.) antennas were designed to use laser interferometers to detect the G.W. signal at the frequencies from $0.1 \mathrm{mHz}$ to $1 \mathrm{~Hz}$, and the corresponding working baselines for those interferometers were ranging from a few hundred thousand kilometers to several million kilometers [1-8]. After long distance transmission, only a small fraction of the transmitted laser beam sent by the local satellite can be received by the detector of the remote satellite because of the large beam divergence and the finite aperture of the telescope $[9,10]$. With a Gaussian beam, the received laser power $P_{\mathrm{r}}$ can be shown as [11]

$\dagger$ These authors contributed equally to this work

*Corresponding author (email: gajin@imech.ac.cn)

$$
P_{\mathrm{r}}=0.50 \frac{D^{4}}{\lambda^{2} L^{2}} P
$$

where $P$ is the transmitted laser power, $D$ the diameter of the telescope, $L$ the arm length and $\lambda$ the wavelength of the light. For example, the transmitted light power is $1 \mathrm{~W}$ for laser interferometer space antenna (LISA) $[1,12]$, the diameter of the telescope is $40 \mathrm{~cm}$, the arm length of the interferometer is 5 million $\mathrm{km}$ and the laser wavelength is 1064 $\mathrm{nm}$. As a result, the received laser power by the photodiode of the remote satellite is about $100 \mathrm{pW}$. Supposing the laser is directly reflected from the remote satellite, we can estimate the round-trip power attenuation is about $10^{-20}$ and the final received power of the local satellite is only about 10 $\mathrm{zW}$, which is extremely difficult to detect interferometrically. A technique called 'weak-light phase-locking' (WLPL) [13-15] is introduced into the remote satellite and makes it a 'phase-transponder', as shown in Figure 1. The 
transmitting light is no longer simply reflected by the remote satellite, but interfered and phase-locked with the slave laser. Consequently, the high power slave laser carrying the complete phase information of the transmitting light is sent back to the local satellite. After the laser is finally received by the local satellite, the phase is detected and recorded to derive the passing gravitational waves by post-processing on ground. Although WLPL is unnecessary in the ground based laser interferometric gravitational wave observers, it has been researched along with the increasing interest in weak light detection and the thriving scheme of extremely long laser ranging in space by scientists since the end of the last century. In previous experimental demonstration of WLPL for LISA, the laser measurement instrument noises, however, have not been considered [13-20]. Since the phase-locking at pico-watt region is very stringent due to the low signal to noise ratio (SNR), the systematic complicity and the technical difficulty will make the situation even worse in debt of the introduction of such the instrument noises as shot noise, pointing jitter noise, laser frequency noise, clock jitter noise, phase readout noise, proof mass acceleration noise, etc.. Thus, it is necessary to perform a comprehensive simulation of WLPL for LISA to better understand beforehand the effects and influences induced by additional noises.

In section 2 the major noise sources are briefly reviewed. Conventionally, all the noises are given in amplitude spectral density (ASD) and approximated by a smooth function of frequency. In order to convert the noise function into real time data with random characteristics, an algorism based on Fourier transform is introduced in section 3. Finally, the simulation of WLPL for LISA and the results are presented in section 4 .

\section{Investigation of noises}

To simulate WLPL for LISA in a systematic manner, the noise sources categorized have to be taken into account as

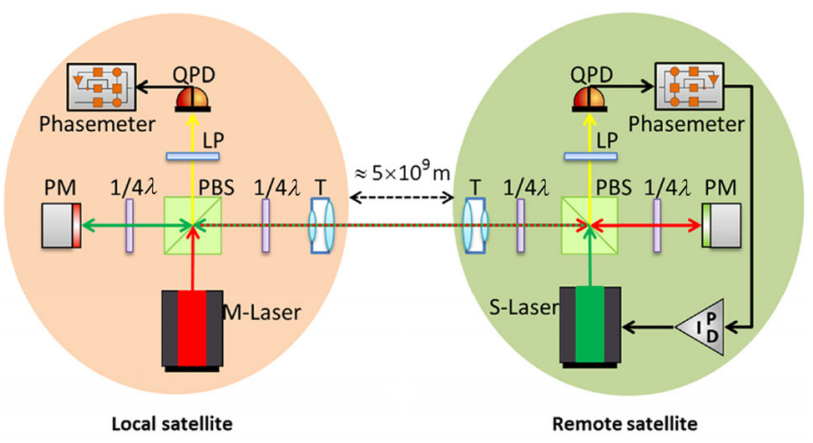

Figure 1 (Color online) Schematic diagram of WLPL scheme for LISA. LP: linear polarizer; M: laser-master laser, PBS: polarizing beam splitter; PM: proof mass; QPD: quadrant photo detector; S-laser: slave laser; T: telescope. the interferometric measurement system (IMS) noise and the acceleration noise. For the interests of LISA, only the frequency domain from $0.1 \mathrm{mHz}$ to $1 \mathrm{~Hz}$ is considered.

\subsection{IMS noise}

The performance of LISA will be dominated by the IMS noise, such as shot noise, laser pointing jitter noise, laser frequency noise, clock jitter noise, etc., which will be discussed in the subsequent sections. Since the initial laser frequency noise and clock jitter noise are several orders of magnitude larger than LISA requirement, they need to be eliminated by various invented technics and need more careful consideration.

\subsubsection{Shot noise}

Shot noise is mainly limited by the distance between two satellites and the finite aperture of the telescopes, which has a sizeable contribution to the IMS noise $[1,11]$. The displacement noise equivalent from shot noise is denoted by [11]

$$
\delta L=\sqrt{\frac{\hbar c}{2 \pi} \cdot \frac{\lambda}{P_{\text {avail }}}},
$$

where $\hbar$ is the reduced Plank constant, $c$ the speed of light in vacuum, $\lambda$ the wavelength of laser and $P_{\text {avail }}$ available light power. For LISA, the available power of the incoming light is roughly $100 \mathrm{pW}$, resulting in the displacement noise due to shot noise about $7.7 \mathrm{pm} / \sqrt{\mathrm{Hz}}$ in the frequency band from $0.1 \mathrm{mHz}$ to $1 \mathrm{~Hz}$ [1].

A small part of the slave laser around $200 \mu \mathrm{W}$ is used as the oscillator interfering with the received light and the equivalent displacement noise due to the oscillator shot noise is on the order of $1 \mathrm{fm} / \sqrt{\mathrm{Hz}}$, which can be ignored.

\subsubsection{Beam pointing jitter noise}

Disturbed by the residual non-conservative forces, the angular jitter of the transmitting laser will cause displacement noise $[21,22]$. Beam pointing control system $[23,24]$ is constructed to improve the pointing stability and suppress the pointing noise to $5.3 \mathrm{pm} / \sqrt{\mathrm{Hz}} \cdot \sqrt{1+(2.8 \mathrm{mHz} / f)^{4}}$ in the frequency range of $0.1 \mathrm{mHz}$ to $1 \mathrm{~Hz}$ [1].

\subsubsection{Laser frequency noise}

To satisfy LISA's requirement, laser frequency noise has to be suppressed to lower than $2 \mathrm{pm} / \sqrt{\mathrm{Hz}}$ $\cdot \sqrt{1+(2.8 \mathrm{mHz} / f)^{4}} \quad$ [1]. While the free running laser frequency stability $\delta f$ is around $10^{4} \mathrm{~Hz} / f \cdot \mathrm{Hz} / \sqrt{\mathrm{Hz}}$, and it can be transfered to the displacement noise $\delta L$ by the following formula $[1,25]$ : 


$$
\delta L=\frac{\delta f}{f_{1}} \Delta L
$$

where $f$ is the measurement frequency, $f_{1}$ the laser frequency, and $\Delta L$ the difference of optical pathlength between two interfering lights.

From eq. (3), the free-running laser frequency noise is $0.17 \mathrm{~Hz} / f \cdot \mathrm{m} / \sqrt{\mathrm{Hz}}$, which is several orders of magnitude greater than the requirement of LISA. Therefore, a series of approaches are proposed to suppress the frequency noise: pre-stabilization, arm-locking and a post-processing method called time-delay interferometry (TDI) [1,26]. By pound-drever-hall $(\mathrm{PDH})$ pre-stabilization, the frequency stability can be achieved $30 \mathrm{~Hz} / \sqrt{\mathrm{Hz}} \cdot \sqrt{1+(2.8 \mathrm{mHz} / f)^{4}}$, which means the frequency noise can be suppressed to $5 \times 10^{-4} \mathrm{~m} / \sqrt{\mathrm{Hz}} \cdot \sqrt{1+(2.8 \mathrm{mHz} / f)^{4}} \quad[27,28]$. Furthermore, the arm-locking technique can improve the frequency stability for two orders, and the corresponding frequency noise is down to $5 \times 10^{-6} \mathrm{~m} / \sqrt{\mathrm{Hz}} \cdot \sqrt{1+(2.8 \mathrm{mHz} / f)^{4}} \quad[1,29,30]$. At last, TDI brings the frequency noise to $2 \mathrm{pm} / \sqrt{\mathrm{Hz}} \cdot \sqrt{1+(2.8 \mathrm{mHz} / f)^{4}}$, which meets LISA's requirement [1,31-33].

In the simulation, the effects of upper four different stages to WLPL will be highlighted.

\subsubsection{Clock jitter noise}

The clock noise is another essential element, because the space-qualified ultra-stable oscillator (USO) cannot meet LISA's requirement $[34,35]$. The relationship between the clock noise and the stability of the USO is $[33,36]$ :

$$
\delta L=\Delta f \cdot \delta t \cdot \lambda,
$$

where $\Delta f$ is the heterodyne frequency, $\delta t$ the stability of the USO and $\lambda$ the wavelength. The free-running stability of the USO is $7 \times 10^{-14} \mathrm{~Hz} / f \cdot \mathrm{s} / \sqrt{\mathrm{Hz}}$, causing the free-running clock noise $1.49 \mathrm{~Hz} / f \cdot \mathrm{pm} / \sqrt{\mathrm{Hz}}$ in the frequency domain from $0.1 \mathrm{mHz}$ to $1 \mathrm{~Hz}$, which cannot achieve $2 \mathrm{pm} / \sqrt{\mathrm{Hz}} \cdot \sqrt{1+(2.8 \mathrm{mHz} / f)^{4}}$ especially in the low-frequencies around $1 \mathrm{mHz}[1,36]$.

\subsubsection{Readout noise}

Consists of the photodiode error [37] and the phasemeter noise [38-40], the readout noise is required to achieve $3.16 \mathrm{pm} / \sqrt{\mathrm{Hz}} \cdot \sqrt{1+(2.8 \mathrm{mHz} / f)^{4}}$ in the measurement band of LISA [1].

\subsubsection{Residual IMS noise}

In addition to the above five noise sources, the interferometric measurement system still has some vital noise sources, such as intensity noise, telescope pathlength noise, optical bench pathlength noise, etc. In the following experiments, the residual IMS noise is considered as a whole, whose ASD is $5.6 \mathrm{pm} / \sqrt{\mathrm{Hz}} \cdot \sqrt{1+(2.8 \mathrm{mHz} / f)^{4}}$ in the frequency band between $0.1 \mathrm{mHz}$ and $1 \mathrm{~Hz}$ [1].

\subsection{Acceleration noise}

To sense and detect the gravitational wave, the proof-mass have to follow a free-falling trajectory. Therefore, the disturbance reduction system is used to shield the proof-mass from the various disturbances. However, the residual effect will still dominate the LISA's performance in the frequencies lower than $1 \mathrm{mHz}$. The ASD for the residual acceleration $\delta a$ without margin on the proof-mass is $1.95 \times 10^{-15}$ $\mathrm{m} / \mathrm{s}^{2} / \sqrt{\mathrm{Hz}} \cdot \sqrt{1+(f / 8 \mathrm{mHz})^{4}} \cdot \sqrt{1+(0.1 \mathrm{mHz} / f)} \quad$ [1]. Therefore, the equivalent displacement noise is shown in [1]:

$$
\begin{aligned}
\delta L & =2 \times \frac{\delta a}{(2 \pi f)^{2}} \\
& =1.54 \mathrm{pm} / \sqrt{\mathrm{Hz}} \cdot \sqrt{1+(8 \mathrm{mHz} / f)^{4}} \cdot \sqrt{1+(0.1 \mathrm{mHz} / f)} .
\end{aligned}
$$

\section{Generating the noise in time domain}

Since the simulation will be run in time series, the above LISA noises given by smooth function in ASD should be converted into random noise in time domain. Besides, the random characteristics of the LISA noises have been smoothed out since they were approximated by the above noise functions. To recover the randomness for the LISA noises in time domain, a dimensionless band-limited Gaussian white noise is generated in the frequency range from $0.1 \mathrm{mHz}$ to $1 \mathrm{~Hz}$. The linear fitting of the ASD of the Gaussian white noise will be normalized to $1 / \sqrt{\mathrm{Hz}}$. Meanwhile, a symmetrical two-side LISA noise ASD functions is created by using the above one-side ASD functions. Then we multiply the normalized Gaussian white noise ASD by the two-side LISA noise ASD. Finally, the multiplication data will be inverse Fourier transformed to obtain the desired LISA noises in time domain.

To verify the validity of the generated LISA noises in time domain, the one-side ASD have been calculated and compared with the original one-side ASD functions given in section 2. Three examples are given in Figure 2. In Figure 2(a), the original ASD function of the IMS noise with margin $18 \mathrm{pm} / \sqrt{\mathrm{Hz}} \cdot \sqrt{1+(2.8 \mathrm{mHz} / f)^{4}}$ is plotted in bold black line, where the blue dotted line is the ASD of generated the IMS noise. The original ASD function of the acceleration noise with margin $2.36 \mathrm{pm} / \sqrt{\mathrm{Hz}} \cdot \sqrt{1+(8 \mathrm{mHz} / f)^{4}}$ $\cdot \sqrt{1+(0.1 \mathrm{mHz} / f)}$ is plotted in Figure 2(b) in bold black 

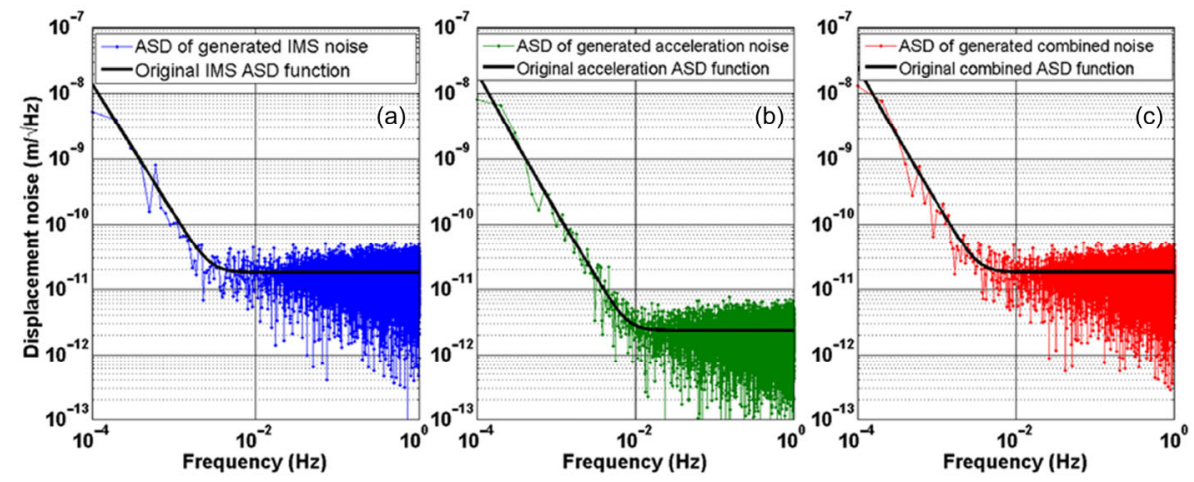

Figure 2 (Color online) Comparison of the original ASD functions and the ASD of corresponding generated noises of the IMS noise (a), the acceleration noise (b) and the combined noise (c).

line, and the ASD of generated acceleration noise is given in green dotted line. The ASD function of original combined noise $\sqrt{\left(\delta L_{\mathrm{IMS}}\right)^{2}+\left(\delta L_{\text {Acceleration }}\right)^{2}}$ is given in bold black line in Figure 2(c) and the red dotted line is the ASD of generated combined noise. As shown in the figure, the generated time-domain data can well represent the corresponding original noise functions.

\section{Simulation, results and discussion}

Based on Figure 1, a simulation program using Matlab Simulink is established and the schematic diagram of the program is shown in Figure 3. The transmitting light received by the remote satellite is represented by 'add1' in which all the noises have been contained. A single frequency 'G.W. signal' ( $100 \mathrm{pm} / \sqrt{\mathrm{Hz}}$ at $30 \mathrm{mHz}$ ) is also added as an indicator to analyze the impacts of various noises. The slave laser in the remote satellite is given as 'add2' where only shot noise is concerned. The slave laser and the received light "interfere" at 'add3' and the phase difference is sent to and read out by a phasemeter which is represented by 'add4'. The readout noise contains additional noise come from photo detector, ADC, transmitting wire and phasemeter. Then, the PID controller is introduced to feedback control the slave laser to phase-locking the transmitting light. For later analysis, the phase of received light is plotted from port ' $x 1$ ', the phase of slave laser will be read from port ' $x 2$ '. The in-loop data used as error function sent to PID controller could be read out from port ' $x 3$ '. Port ' $x 4$ ' gives the out-of-loop signal which indicates the phase deviation between the slave laser and the received light after the loop is closed. Thus the out-of-loop signal could be seen as the additional noise due to the phase-locking loop. The Bode diagram of the PID controller is shown in Figure 4.

The addition of the 'G.W. signal' is given based on the references [41-43] that the strain amplitude of major LISA G.W. sources, after one year integration, could reach to a level from $10^{-21} / \sqrt{\mathrm{Hz}}$ to $10^{-18} / \sqrt{\mathrm{Hz}}$ strongly depends

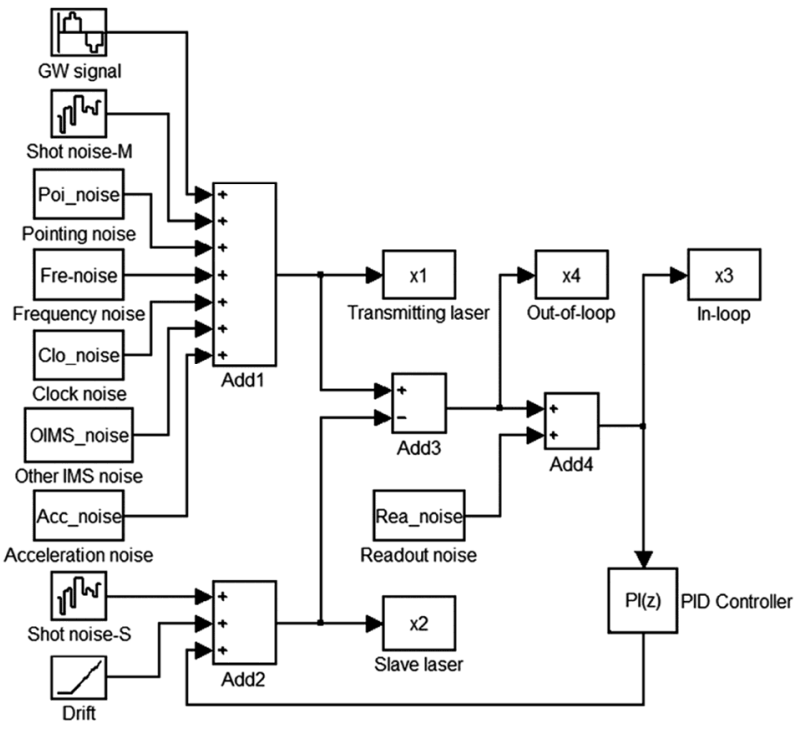

Figure 3 The Simulink model of WLPL arrangement for LISA.

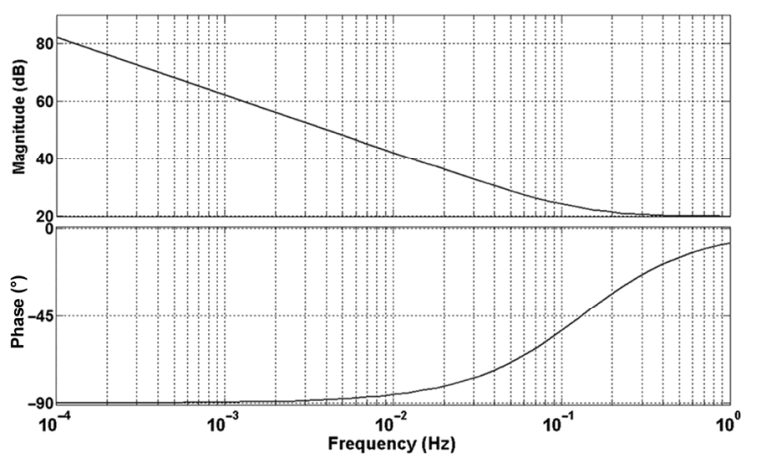

Figure 4 Bode diagram of the control system.

on the distances and types.

In the following sections, the effects of the frequency noise and the clock noise on the performance of WLPL for LISA will be emphasized. In the first three experiments, the clock noise is free-running and the frequency noise is under such different suppression schemes as free-running, pre- 
stabilization and arm-locking. In the latter two experiments, all the IMS noise and the acceleration noise have been satisfied LISA's requirement without and with margin respectively. The phase of the transmitting light, the phase of slave laser, the in-loop signal and the out-of-loop signal in close-loop are analyzed by ASD and shown in blue, green, red and aquamarine, individually. As a comparison, the required LISA noise floor is given in bold black.

\subsection{Case 1: Free-running frequency noise and clock noise}

In this case, the laser frequency noise and the clock jitter noise are not suppressed and the laser frequency noise dominates the noise performance. As shown in Figure 5, the ASD of the phase noise of the slave laser is almost the same as the transmitting light and reaches $10^{2} \mathrm{~m} / \sqrt{\mathrm{Hz}}$ at the frequencies around $1 \mathrm{mHz}$. Moreover, the ASD of the outof-loop signal is also similar to the in-loop signal since the readout noise can be ignored compared to the in-loop signal.

\subsection{Case 2: Pre-stabilized frequency noise and free- running clock noise}

The laser frequency is pre-stabilized by PDH method while the clock jitter noise is still free running. The ASD of the slave laser still coincides with the one of the transmitting light, as shown in Figure 6. Apparently the laser frequency noise is improved for at least 4 orders because of the pre-stabilization. Meanwhile, the out-of-loop signal representing the deviated phase between the slave oscillator and the transmitting light is also improved for several orders.

\subsection{Case 3: Arm-locking frequency noise and free- running clock noise}

In case 3, the laser frequency noise has been further suppressed by using arm-locking technique and the clock jitter noise is still let free (Figure 7). The laser frequency noise is still dominating, however it is suppressed for about two orders (section 2). The precision of the locking scheme represented by the out-of-loop signal is now about $10^{-7} \mathrm{~m} / \sqrt{\mathrm{Hz}}$.

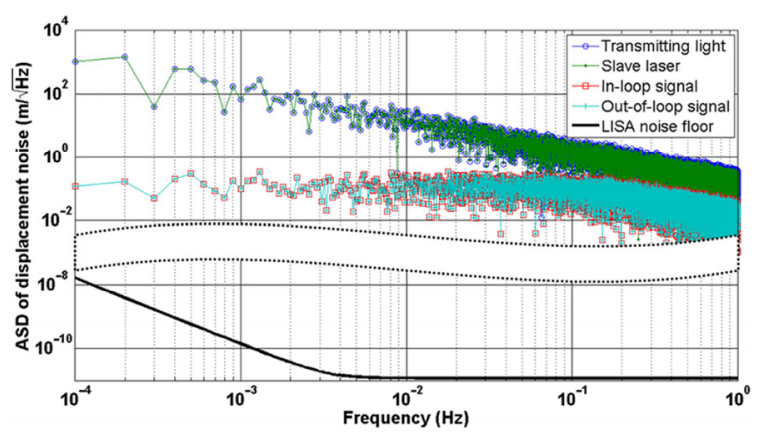

Figure 5 ASD noise performance in case 1.

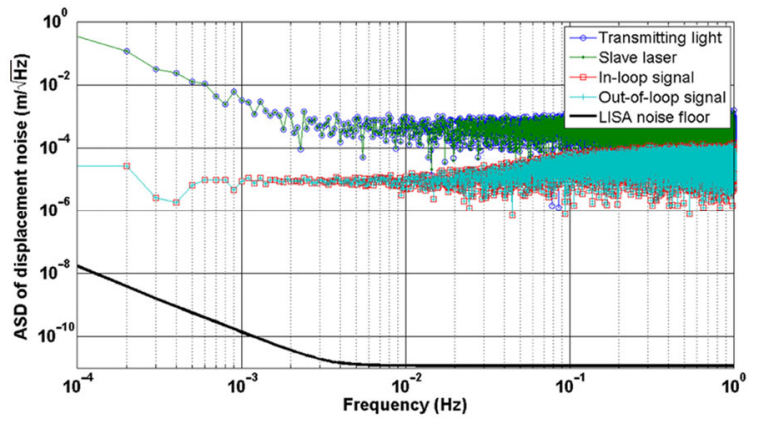

Figure 6 ASD noise performance in case 2.

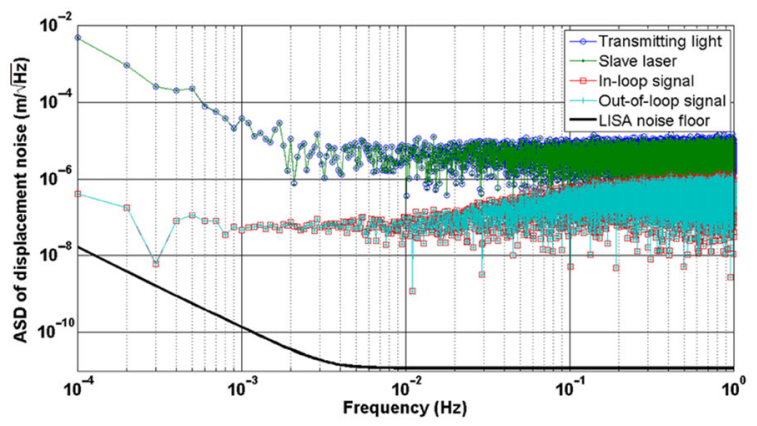

Figure 7 ASD noise performance in case 3.

\subsection{Case 4: TDI noise without margin}

In this case, the frequency noise and the clock jitter noise have been well suppressed by TDI and other post process technics. Therefore, the WLPL performance is dominated not only by the laser frequency noise but jointly by the rest IMS noise and the acceleration noise. Again, the phase of the slave laser and the phase of the transmitting light well coincide with each other (Figure 8). It could be seen, the out-of-loop signal is mainly limited by the readout noise (shown in the black dotted line) and the in-loop signal now is well below $10^{-12} \mathrm{~m} / \sqrt{\mathrm{Hz}}$. Under this situation, the 'G.W. signal' is about to emerge out.

\subsection{Case 5: TDI noise with margin}

Considering the contingency in science measurement, the

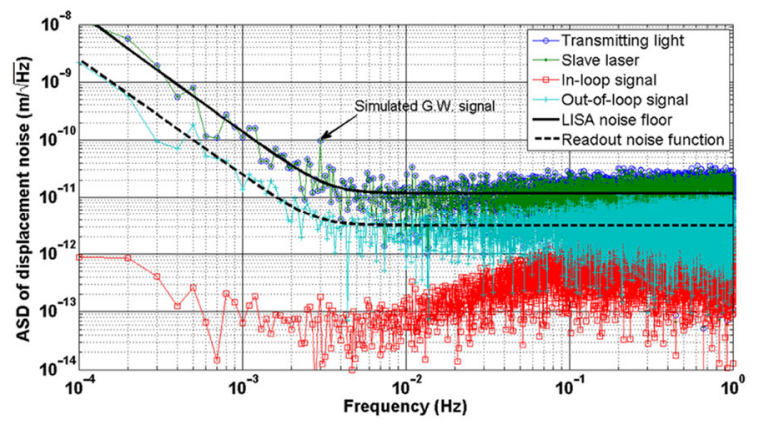

Figure 8 ASD noise performance in case 4. 
noise budget should include adequate margin. The official total IMS noise with margin is $18 \mathrm{pm} / \sqrt{\mathrm{Hz}}$ $\cdot \sqrt{1+(2.8 \mathrm{mHz} / f)^{4}}$ and the official acceleration noise with margin is about $3.0 \times 10^{-15} \mathrm{~m} / \mathrm{s}^{2} / \sqrt{\mathrm{Hz}} \cdot \sqrt{1+(f / 8 \mathrm{mHz})^{4}}$ $\cdot \sqrt{1+(0.1 \mathrm{mHz} / f)}$ in the frequency band between $0.1 \mathrm{mHz}$ and $1 \mathrm{~Hz}$. The LISA noise floor given in bold black line is plotted based on the previous noises with margin. The ASD of the four signals from $x 1$ to $x 4$ is plotted in Figure 9. Compared to the four signals in case 4 , the values of the four signal in case 5 are about sixty percent greater respectively.

\subsection{Benchtop test and discussion}

An experimental demonstration is also performed in order to test the control scheme. The slave laser is interfered with the master laser on a benchtop interferometer with a beat frequency of $1 \mathrm{MHz}$. The beat signal is then sent to a phasemeter where the phase difference between the slave laser and the master laser is read out. As the error function, the phase difference data is sent to the controller to adjust the phase of the slave laser to track along with the phase of the master laser. The schematic diagram and the result of the experimental test are given in Figure 10.

Limited by the readout noise and optical pathlength noise (one of the residual IMS noise), the phase lock loop is working at nanometer level. The power of the slave laser and the master laser are about $1 \mathrm{~mW}$ that the shot noise could be ignored. It could be seen that the phase difference between the slave laser and the master laser is suppressed from $10^{-7}$ to $3 \times 10^{-10} \mathrm{~m} / \sqrt{\mathrm{Hz}}$ at $1 \mathrm{mHz}$ after the phase locking loop is turned on. This gives a direct proof of the

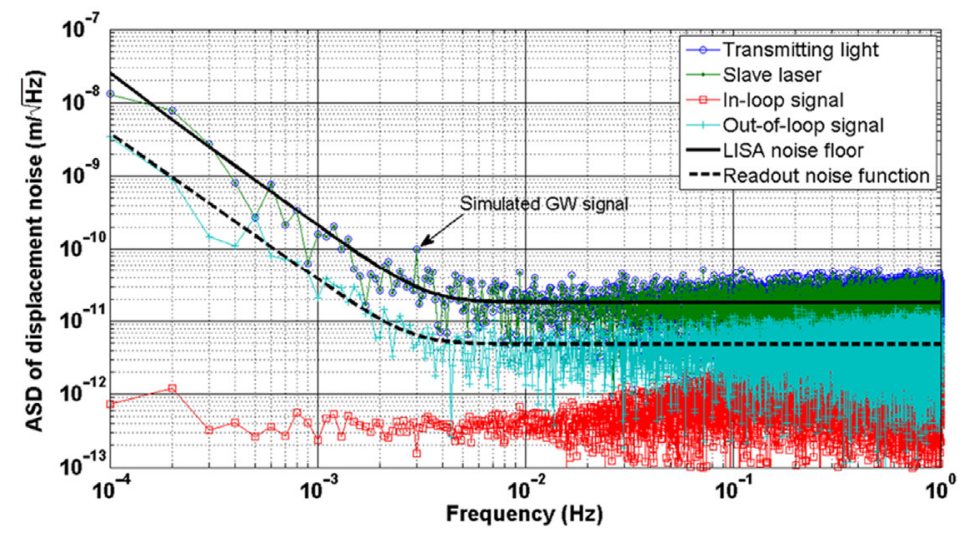

Figure 9 ASD noise performance in case 5.
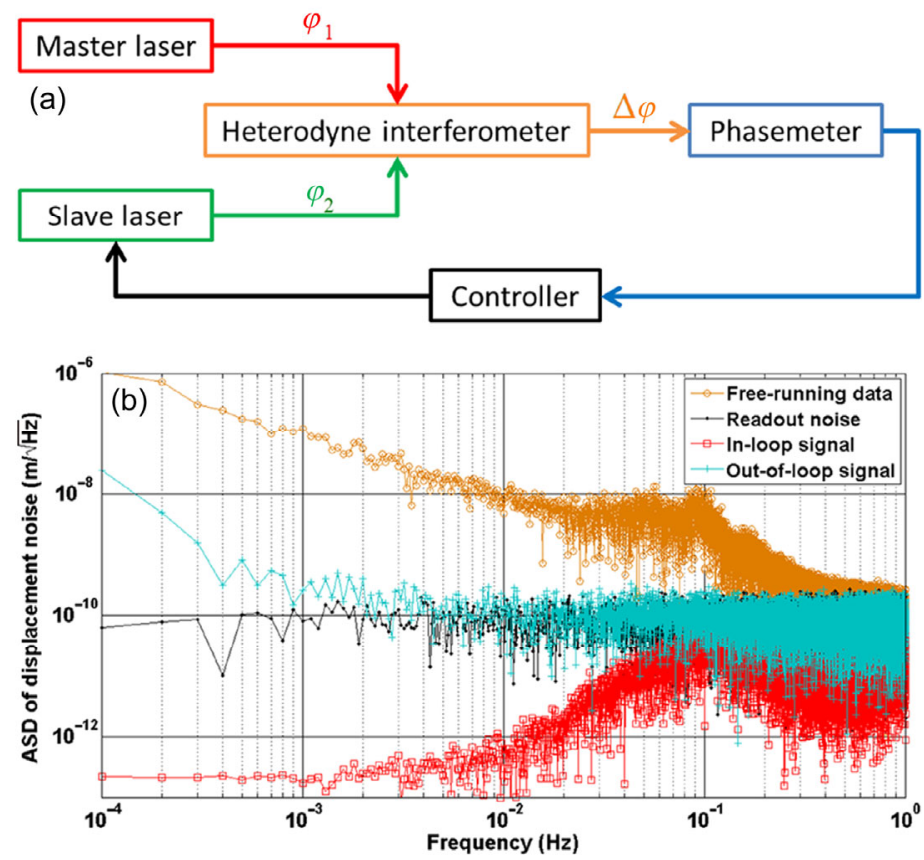

Figure 10 Schematic diagram (a) and result of the experimental test (b). 
functionality of our control scheme.

The locking precision represented by the out-of-loop data is limited by the readout noise at higher frequencies, and in the lower frequencies is believed to be limited by the optical pathlength noise caused by thermal expansion of the interferometer. Though the amplitude and the frequency characteristics of the noise in the testing experiment are very different from those concerned in case 1 to 5, the limitation of locking precision with respect to the IMS noise and the readout noise is of the same manner. It indicates that the software simulation accords well with the hardware verification.

\section{Conclusions and outlook}

In the previous five simulating cases, the phase of the slave laser could be seen well coincide with the phase of the transmitting light. The results prove that the WLPL loop works effectively. The phase difference between the slave laser and the transmitting light is given by the out-of-loop signal which indicates how precise the phase of the slave laser traces the phase of the transmitting light. It could be seen in case 4 and 5 that the noise due to the locking scheme given by the out-of-loop signal is well below the required LISA noise floor and dominated by the readout noise. The emergence of the simulated G.W. signal in case 4 and 5 indicates that a better SNR is apparently helpful for signal detection. For a decent precision of waveform extraction it was believed that the SNR needs to be greater than 8 [44]. In case 5, the noise floor recovered from the simulation (see blue and green dots) fits well with the required LISA noise floor, which proved the validity of our simulation.

Unlike Mars mission [17], the SNR for LISA is much higher. It has been mentioned that Laser Interferometer Space Antenna (LISA) phasemeter looked at reducing the probability of cycle slipping when tracking a $3.5 \mathrm{pW}$ signal [18]. Indeed, the received power for LISA will be more than $100 \mathrm{pW}$, the phase lock loop will operate far outside any cycle slip region and therefore in the linear region [45]. In the following plan, a bench-top pW level WLPL control system with $40 \mathrm{kHz}$ bandwidth will be construct and researched.

This work was supported by the Space Science Research Projects in Advance (Grant No. O930143XM1), and the Scientific Equipment Development and Research Project (Grant No. Y231411YB1) of Chinese Academy of Sciences.

1 Danzmann K, Prince T A, Binetruy P, et al. LISA: Unveiling a hidden Universe. Assessment Study Report ESA/SRE, 2011. 54-72

2 Gong X F, Xu S, Bai S, et al. A scientific case study of an advanced LISA mission. Class Quantum Grav, 2011, 28: 094012

3 Li Y Q, Luo Z R, Liu H S, et al. Laser interferometer used for satellite- satellite tracking: an on-ground methodological demonstration.
Chin Phys Lett, 2012, 29: 079501

4 Danzmann K, Seoane P A, Aoudia S, et al. The Gravitational Universe: A science theme addressed by the eLISA mission observing the entire Universe. eLISA white paper, 2013: 13-15

5 Bender P L, Begelman M C, Gair J R. Possible LISA follow-on mission scientific objectives. Class Quantum Grav, 2013, 30: 165017

6 Ni W T. ASTROD-GW: Overview and progress. Int J Mod Phys D, 2013, 22: 1341004

7 Wang Y, Keitel D, Babak S, et al. Octahedron configuration for a displacement noise-cancelling gravitational wave detector in space. Phys Rev D, 2013, 88: 104021

8 Gong X, Lau Y K, Xu S, et al. Descope of the ALIA mission. J Phys Conf Ser, 2015, 610: 012011

9 Liao A C, Ni W T, Shy J T. Pico-watt and femto-watt weak-light phase locking. Int J Mod Phys D, 2002, 11: 1075-1085

10 Diekmann C, Steier F, Sheard B, et al. Analog phase lock between two lasers at LISA power levels. J Phys Conf Ser, 2009, 154: 012020

11 Danzmann K, Bender P, Brillet A, et al. LISA pre-phase A report. 2nd ed. Max-Planck-Institut fur Quantenoptik Report No. MPQ 208. Garching, Germany, 1998. 57-82

12 Luo Z R, Bai S, Bian X, et al. Space laser interferometry gravitational wave detection (in Chinese). Adv Mech, 2013, 43: 415-447

13 McNamara P W, Ward H, Hough J. Laser phase-locking techniques for LISA: Experimental status. AIP Conf Proc, 1998, 456: 143-147

14 McNamara P W. Weak-light phase locking for LISA. Class Quantum Grav, 2005, 22: S243-S247

15 Esteban J J, García A F, Barke S, et al. Experimental demonstration of weak-light laser ranging and data communication for LISA. Opt Express, 2011, 19: 15937-15946

16 Ni W T, Pan S, Peng G S, et al. Progress in laboratory R \& D for fundamental physics space missions-weak light phase-locking, fibre-linked heterodyne interferometry, fibre delay line and picometre real-time motion control. Class Quantum Grav, 1996, 13: A311A315

17 Dick G J, Tu M, Strekalov M D, et al. Optimal phase lock at femtowatt power levels for coherent optical deep-space transponder. IPN Progress Report, 2008. 42-175

18 Francis S P, Lam T T Y, McKenzie K, et al. Weak-light phase tracking with a low cycle slip rate. Opt Lett, 2014, 39: 5251-5254

19 Mitryk S J, Wand V, Mueller G. Verification of time-delay interferometry techniques using the University of Florida LISA interferometry simulator. Class Quantum Grav, 2010, 27: 084012

20 Shaddock D, Ware B, Halverson P G, et al. Overview of the LISA Phasemeter. AIP Conf Proc, 2006, 873: 654-660

21 Robertson D I, McNamara P, Ward H, et al. Optics for LISA. Class Quantum Grav, 1997, 14: 1575-1577

22 Bender P L. Wavefront distortion and beam pointing for LISA. Class Quantum Grav, 2005, 22: S339-S346

23 Dong Y H, Liu H S, Luo Z R, et al. Methodological demonstration of laser beam pointing control for space gravitational wave detection missions. Rev Sci Instrum, 2014, 85: 074501

24 Dong Y H, Liu H S, Luo Z R, et al. Principle demonstration of fine pointing control system for inter-satellite laser communication. Sci China Tech Sci, 2015, 58: 449-453

25 Jennrich O. LISA technique and instrumention. Class Quantum Grav, 2009, 26: 153001

26 Cruz R J, Thorpe J I, Preston A, et al. The LISA benchtop simulator at the University of Florida. Class Quantum Grav, 2006, 23: S751S760

27 Peterseim M, Brozek O S, Danzmann K, et al. Laser development and laser stabilization for the space-borne gravitational wave. AIP Conf Proc, 1998, 456: 148-155

28 Mueller G, McNamara P, Thorpe I, et al. Laser frequency stabilization for LISA. Technical Memorandum NASA/TM-2005-212794, 2005. 1-17

29 Sheard B S, Gray M B, McClelland D E, et al. Laser frequency stabilization by locking to a LISA arm. Phys Lett A, 2003, 320: 9-21

30 Yu Y, Mitryk S, Mueller G. Arm locking for space-based laser interferometry gravitational wave observatories. Phys Rev D, 2014, 90: 
062005

31 Tinto M, Estabrook F B, Armstrong J W. Time-delay interferometry for LISA. Phys Rev D, 2002, 65: 082003

32 Shaddock D A, Ware B, Spero R E, et al. Postprocessed time-delay interferometry for LISA. Phys Rev D, 2004, 70: 081101

33 de Vine G, Ware B, McKenzie K, et al. Experimental demonstration of time-delay interferometry for the laser interferometer space antenna. Phys Rev Lett, 2010, 104: 211103

34 Heinzel G, Esteban J J, Barke S, et al. Auxiliary functions of the LISA laser link: Ranging, clock noise transfer and data communication. Class Quantum Grav, 2011, 28: 094008

35 Otto M, Heinzel G, Danzmann K. TDI and clock noise removal for the split interferometry configuration of LISA. Class Quantum Grav, 2012, 29: 205003

36 Klipstein W, Halverson P G, Peters R, et al. Clock noise removal in LISA. AIP Conf Proc, 2006, 873: 312-318

37 Cervantes F G, Livas J, Silverberg R, et al. Characterization of photoreceivers for LISA. Class Quantum Grav, 2011, 28: 094010

38 Liu H S, Dong Y H, Li Y Q, et al. The evaluation of phasemeter pro- totype performance for the space gravitational waves detection. Rev Sci Instrum, 2014, 85: 024503

39 Liang Y R, Duan H Z, Yeh H C, et al. Fundamental limits on the digital phase measurement method based on cross-correlation analysis. Rev Sci Instrum, 2012, 83: 095110

40 Liu H S, Dong Y H, Luo Z R, et al. Multi-channel phasemeter and its application in the heterodyne laser interferometry. Sci China Tech Sci, 2015, 58: 746-749

41 Sathyaprakash B S, Schutz B F. Physics, astrophysics and cosmology with gravitational waves. Living Rev Relativ, 2009, 12: 1

42 Camp J B, Cornish N J. Gravitational wave astronomy. Annu Rev Nucl Part Sci, 2004, 54: 525-577

43 Moore C J, Cole R H, Berry C P L. Gravitational-wave sensitivity curves. Class Quantum Grav, 2015, 32: 015014

44 Ajith P. Gravitational-wave data analysis using binary black-hole waveforms. Class Quantum Grav, 2008, 25: 114033

45 Gerberding O, Sheard B, Bykov I, et al. Phasemeter core for intersatellite laser heterodyne interferometry: Modelling, simulations and experiments. Class Quantum Grav, 2013, 30: 235029 